The following is a brief summary only. Before prescribing, see complete prescribing information in ZANTAC* product labeling

INDICATONS ANO USAGE: ZANTAC* is indicated in:

1. Short-term treatment of active duodenal ulcer. Most patients heal within four weeks.

2. Maintenance therapy for duodenal ulcer patients at reduced dosage after healing of acute ulcers.

3. The treatment of pathological hypersecretory conditions (eg. Zollinger-Ellison syndrome and systemic

mastocytosis)

4. Short-term treatment of active, benign gastric ulcer. Most patients heal within six weeks and the

usefulness of further treatment has not been demonstrated

5. Treatment of gastroesophageal reflux disease (GERD). Symptomatic relief commonly occurs within

one or two weeks after starting therapy. Therapy for longer than six weeks has not been studied.

In active duodenal ulcer; active, benign gastric uloer; hypersecretory states; and GERD, concomitant

antacids should be given as needed for relief of pain.

CONTRAINDICATIONS: ZANTAC ${ }^{*}$ is contraindicated for patients known to have hypersensitivity to the drug

PRECAUTIONS: General: 1 . Symptomatic response to ZANTAC` therapy does not preclude the presence

of gastric malignancy.

2. Since ZANTAC is excreted primarily by the kidney, dosage should be adjusted in patients with impaired

renal function (see DOSAGE AND ADMINISTRATION). Caution should be observed in patients with hepatic dysfunction since ZANTAC is metabolized in the liver.

Laboratory Tests: False-positive tests for urine protein with Multistix may occur during ZANTAC therapy

and therefore testing with sulfosalicylic acid is recommended.

Drug Interactions: Although ZANTAC has been reported to bind weakly to cytochrome P-450 in vitro.

recommended doses of the drug do not inhibit the action of the cytochrome P-450-linked oxygenase

enzymes in the liver. However, there have been isolated reports of drug interactions which suggest that

ZANTAC may affect the bioavailability of certain drugs by some mechanism as yet unidentified (eg. a

pH-dependent effect on absorption or a change in volume of distribution).

Carcinogenesis, Mutagenesis, Impairment of Fertility: There was no indication of tumorigenic or

carcinogenic effects in lifespan studies in mice and rats at doses up to $2,000 \mathrm{mg} / \mathrm{kg} / \mathrm{day}$

Ranitidine was not mutagenic in standard bacterial tests (Salmonella, $E$ coli) for mutagenicity at

concentrations up to the maximum recommended for these assays.

In a dominant lethal assay, a single oral dose of $1,000 \mathrm{mg} / \mathrm{kg}$ to male rats was without effect on the

outcome of two matings per week for the next nine weeks.

Pregnancy: Teratogenic EHects: Pregnancy Category B: Reproduction studies have been performed in

rats and rabbits at doses up to 160 times the human dose and have revealed no evidence of impaired

fertility or harm to the fetus due to ZANTAC. There are, however, no adequate and well-controlled studies in pregnant women. Because animal reproduction studies are not always predictive of human response. this drug should be used during pregnancy only if clearly needed.

Nursing Mothers: ZANTAC is secreted in human milk. Caution should be exercised when ZANTAC is

administered to a nursing mother.

Pediatric Use: Safety and effectiveness in children have not been established.

Use in Elderly Patients: Ulcer healing rates in elderly patients ( 65 to 82 years of age) were no different

from those in younger age groups. The incidence rates for adverse events and laboratory abnormalities

were also not different from those seen in other age groups.

ADVERSE REACTIONS: The following have been reported as events in clinical trials or in the routine

management of patients treated with oral ZANTAC*. The relationship to ZANTAC therapy has been unclear

in many cases. Headache, sometimes severe, seems to be related to ZANTAC administration.

Centrai Nervous System: Rarely, malaise, diziness, somnolence, insomnia, and vertigo. Rare cases of

reversible mental confusion, agitation, depression, and hallucinations have been reported, predominantly

in severely ill elderly patients. Rare cases of reversible blurred vision suggestive of a change in accommo-

dation have been reported.

Cardiovascular: Rare reports of tachycardia, bradycardia, and premature ventricular beats

Gastrointestinal: Constipation, diarrhea, nausea/vomiting, and abdominal discomfortpa in

Hepatic: In normal volunteers. SGPT values were increased to at least twice the pretreatment levels in 6 of

12 subjects receiving $100 \mathrm{mg}$ qid IV for seven days, and in 4 of 24 subjects receiving $50 \mathrm{mg}$ qid IV for five days. With oral administration there have been occasional reports of reversible hepatitis, hepatocellular or hepatocanalicular or mixed, with or without jaundice.

Musculoskeletal: Rare reports of arthralgias.

Hematologic: Reversible blood count changes (leukopenia, granulocytopenia, thrombocytopenia) have occurred in a few patients. Rare cases of agranulocytosis or of pancytopenia, sometimes with marrow hypoplasia, have been reported.

Endocrine: Controlled studies in animals and man have shown no stimulation of any pituitary hormone by ZANTAC (ranitidine hydrochloride) and no antiandrogenic activity, and cimetidine-induced gynecomastia and impotence in hypersecretory patients have resolved when ZANTAC has been substituted. However, occasional cases of gynecomastia, impotence, and loss of libido have been reported in male patients receiving ZANTAC, but the incidence did not differ from that in the general population.

Integumentary: Rash, including rare cases suggestive of mild erythema multiforme, and rarely, alopecia. Other: Rare cases of hypersensitivity reactions (eg, bronchospasm, fever, rash, eosinophilia) and small increases in serum creatinine.

OVERDOSAGE: Information concerning possible overdosage and its treatment appears in the full prescribing information

DOSAGE AND ADMINISTRATION: Active Duodenal Ulcer: The current recommended adult oral dosage is $150 \mathrm{mg}$ twice daily. An alternate dosage of $300 \mathrm{mg}$ once daily at bedtime can be used for patients in whom dosing convenience is important. The advantages of one treatment regimen compared to the other in particular patient population have yet to be demonstrated.

Maintenance Therapy: The current recommended aduit oral dosage is $150 \mathrm{mg}$ at bedtime.

Pathological Hypersecretory Conditions (such as Zollinger-Ellison syndrome): The current recom-

mended adult oral dosage is $150 \mathrm{mg}$ twice a day. In some patients it may be necessary to administer

ZANTAC 150-mg doses more frequently. Doses should be adjusted to individual patient needs, and should continue as long as clinically indicated. Doses up to $6 \mathrm{~g} / d$ ay have been employed in patients with severe

disease.

Benign Gastric Ulcer: The current recommended adult oral dosage is $150 \mathrm{mg}$ twice a day.

GERD: The current recommended adult oral dosage is $150 \mathrm{mg}$ twice a day.

Dosage Adjustment for Patients with Impaired Renal Function: On the basis of experience with a group of subjects with severely impaired renal function treated with ZANTAC, the recommended dosage in patients with a creatinine clearance less than $50 \mathrm{~m} / \mathrm{min}$ is $150 \mathrm{mg}$ every 24 hours. Should the patient's condition require, the frequency of dosing may be increased to every 12 hours or even further with caution. Hemodialysis reduces the level of circulating ranitidine. Ideally, the dosage schedule should be adjusted so that the timing of a scheduled dose coincides with the end of hemodialysis.

HOW SUPPLIED: ZANTAC* 300 Tablets (ranitidine hydrochloride equivalent to $300 \mathrm{mg}$ of ranitidine) are

yellow, capsule-shaped tablets embossed with "ZANTAC 300" on one side and "Glaxo" on the other. They are available in bottles of 30 (NDC 0173-0393-40) and unit dose packs of 100 tablets (NDC 0173-0393-47)

ZANTAC 150 Tablets (ranitidine hydrochloride equivalent to $150 \mathrm{mg}$ of ranitidine) are white tablets embossed with "ZANTAC 150" on one side and "Glaxo" on the other. They are available in bottles of 60 tablets (NDC 0173-0344-42) and unit dose packs of 100 tablets (NDC 0173-0344-47)

Store between $15^{\circ}$ and $30^{\circ} \mathrm{C}\left(59^{\circ}\right.$ and $\left.86^{\circ} \mathrm{F}\right)$ in a dry place. Protect from light. Replace cap securely after each opening.

\section{Quality control for in-office and independent laboratories}

How confident can you be that the results of your patient's Pap smear are accurate? Not very, if the testimony before the Senate Committee on Labor and Human Resources is to be believed. In a recent session before the committee, Robert Hasselbrack, MD, chief of staff and laboratory director of the Fifth Avenue Medical Center in Seattle, testified that up to $55 \%$ of Pap smears yield false negative results for cervical dysplasia or cancer. At least half of these are attributable to physician sampling errors.

Prompted by media exposure concerning the lack of quality control among all laboratory testing (especially Pap smears) and subsequent inaccurate results, the Health Care Financing Administration is developing new guidelines in this area. As reported in the May $1988 \mathrm{DO}$, these will emphasize quality control, strengthen the inspection process, and upgrade personnel requirements. Scheduled for inclusion in a uniform proficiency testing program to be developed by the Centers for Disease Control are the following: interstate laboratories; laboratories that service Medicare patients in hospitals, nursing homes, and ambulatory surgical centers; and physician office laboratories and health maintenance organizations that perform more than 5,000 tests annually.

Stricter laboratory testing regulations will have significant impact upon physician offices. Estimates show more than 100,000 in-office laboratories perform nearly $50 \%$ of all testing.

In light of these statistics and accompanying allegations, we physicians must improve our in-office testing procedures and sampling techniques. Good quality control and carefully performing all tests will have an immediate positive effect on all results.

THOMAS WESLEY ALLEN, DO, FACOI

\title{
Glaxo
}




\section{Feldene

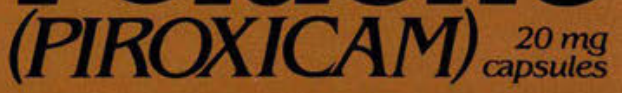

Source: PDR, 1987, Manufacturers' Prescribing Informe Indicates maximum dosage for osteoarthritis and rheumatoid arthritis for the antiarthritic agents shown. Consult manufacturers' prescribing information.

Brief SUmmary
FELOEME: (piroricam) Capsules

CONTRAINDICATIONS: FELDENE (piroxicam) should not be used in patients who have previously exhibited hypersensitivity to it, or in individuals with the syndrome comprised of bronchospasm, nasal polyps and angioedema precipitated by aspirin or other nonsteroidal anti-intlammatory drugs

WARKIMGS: Peptic ulceration, perforation and G.I. bleeding - sometimes severe, and, in some instances fatal - have been reported with patients receiving FELDENE. If FELDENE must be given to patients with a history of upper gastrointestina ract disease, the patient snouio be under close supervision (see AOVERSE REACTIONS)

PRECAUINOMS: Renal Effects: As with other nonsteroidai anti-inflammatory drugs, long-term administration of piroxicam: to animais nas resulted in renal papiliary necrosis and other abnormal renal pathology in human s.
of acute interstitial nephritis with hematuria. proteinuria, and occasionally. nephrotic syndrome

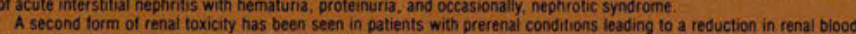
flow or blood volume, where the renal prostaglandins have a supportive role in the maintenance of renal perfusion. in these patients administration of an NSAID may cause a dose-dependent reduction in prostaglandin formation and may precipitate overt renat decompensation. Patients at greatest risk of this reaction are those with impaired renal function, heart failure, iver dystunction, those taking diuretics, and the elderty

Because of extensive renal excretion of piroxicam and its biotranstormation products (less than $5 \%$ of the daily dose excreted unchanged), lower doses of piroxicam should be anticipated in patients with impared renal function. and they

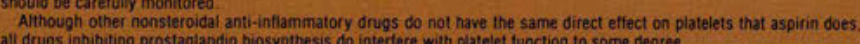
All drugs inhibiting prostaglandin biosynthesis do interfere with platelet function to some degree

Because of reports of adverse eye findings with nonsteroidal anti-inlaammatory agents, it is recommended that patients who develop visual complaints during treatment with FEL DENE have ophthalmic evaluation

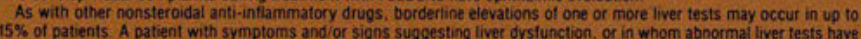
occurred, should be evaluated for evidence of the development of more severe hepatic reaction while on therapy with FELDENE

Severe hepatic reactions, Including jaundice and cases of fatai hepatis have been teported with FELDENE. Although such reactions are rare, If abnormal fiver tests persist or worsen, it clinical signs and symptoms consistent with liver disease develop, of it systemic manifestations occur le,g., eosinophilia, rash, etc.). FELDENE should be discontinued. (See

aiso ADVERSE REACTIONS) did not occur,

Peripheral edema has been observed in approximately $2 \%$ of the patients treated with FELDENE. Theretore. FELDENE A combination of dermatological andith allernic sions and symnton or other conditions predisposing to flivid retention. A combination of dermatological and/or allergic signs and symptoms suggestive of serum sickness have occasionally
occurred in conjunction with the use of fELDENE. These include arthralgias, pruritus, fever, tatigue, and rash including vesiculo buillous reactions and extoliative dermatitis

DRUG INTERACTIONS: Interactions with coumarin-type anticoagulants have been reported with fELDENE since marketing Therefore, physicians should closely monitor patients tor a change in dosage requirements when administering FELDERE to patients on coumarin-type anticoagulants and other highly protein-bound drug:

Plasma levels of piroxicam are depressed 10 approximately $80 \%$ of their normal values when FEL DENE is administered in conjunction with aspirin ( $3900 \mathrm{mg}$ day). but concomitant administration of antacids has no effect on piroxicam plasma

Nonsteroidal ant-inflammatory agents, including FELDENE, have been reported to increase steady state plasma lithium evels. It is recommended that plasma lithium levels be monitored when initiating, adjusting and discontinuing FELDENE.
Carcinogenesis, Chronic Animal Toricity and Impairment of fertility. Subacule and chronic toxicity studies have been Carcinopenesis, Chronic Animal Toxicity and Impairment of Fertility. Subacule and chronic toxicity studies have been The out in rats, mice, dogs, and monkeys:

The pathology most often seen was that characteristically associated with the animal toxicology of anti-inflammatory In classical studies in laboratory animals, pirovicam and gastrointestinal lesions

Reproductive studies revealed no impairment ol tertility in animals.

Pregnancy and Nursing Mothers: Like other drugs which inhibin the synthesis and release of prostaglandins, piroxicam administration continued late into pregnancy increased the incidence of dystocia and delayed parturition in animals. Gastrointestinal tract toxicity was increased in pregnant ternales in the last trimester of pregnancy compared to non-pregnant emales or females in earlier trimesters of pregnancy

FELDENE is not recommended for use in nursing mothers of in pregnant women because of the animal findings and since afety for such use has not been established in humans

Use in Children: Dosage recommendations and indications for use in children have not been established.
UDEFE PEACTiovs

AOVERSE REACTIONS: Gastrointestinal symptoms are the most prominent side effects, occurring in approximately $20 \%$ intestinal side effects, approximately 5\% discontinued therapy with an overail incidence of peptic ulceration of ahout $1 \%$
Adverse reactions are listed beiow by body system for all patients in clinical trials with fELOENE (piroxicam) at of $20 \mathrm{mg} / \mathrm{day}$.

Iacidence Greater Than 1\%: The following adverse reactions occurred more frequently than 1 in 100 Gastrontestinat: stomatitis, anorexia, epigastric distress; nausea: constipation, abdominal discomfort, flatuler archea, abdominal pain, indigestion

tematological decreases in hemoglobin" and hematocrit" (see PRECAUTIONS), anemia, leucopenia, eosinophi' Dermarologic pruritus, rash

Central Nervous System dizziness, somnolence, vertigo

Urogenital: Bun and creatinine eleva:

Soecial Senses tinnitus

Cardiovascular: Respiratory edema (see PRECAUTIONS)

"Reactions occurring in $3 \%$ to $9 \%$ of patients treated with FELOENE

Reactions occurring in $1 \%$ 10 $3 \%$ of patients are unmarkec

incidence Less Than 1\% (Causal Relationship Probabie): The following adverse reactions occurred less frequent in 100 The probability exists that there is a causal relationship between FELDENE and these reactions

Gastrointestinat: liver function abnormalities, jaundice, hepatitis (see PRECAUTIONS), vomiting, hematemesis, n gastrointestinal bleeding, pertoration and ulceration (see WARNINGS), dry mouth

Hematologicat thrombocylopenia, Detechial rash, ecchymosis, bone marrow depression including aplastic a

Dermatologic; sweating, erytherna, bruising. desquamation, exfoliative dermatitis, erythema multitorme, toxic mat necrotysis, Sievens-Johnson syndrome, vesiculo bullous reactions, photoallergic skin reactions

Central Nervous System: depression, insomnia, nervousnoss

Urogenital. hematuria, proteinuria, intersttial nephritis, renal falure, hyperkalemia. glomerulitis papilary ner ephrotic syndrome (see PRECAUTIONS)

Body as a Whole: pain (colic), lever, flu-like syndrome (see PRECAUTIONS)

Special Senses: swollen eyes, blurred vision, eye irritations angina

Metabolic: hypoglycemia, hyperglycemia, weight increase, weight decrease

typersensitivity anaphylaxis, bronchospasm, urticaria angioedema, vasculitis, "serum sickness" (see PRECAUT Incidence Less Than $1 \%$ (Causal Relationship Unknown), Other adverse reactions were reported with a frequency Gastrointestinal pausal relat

Dermatoiogic: onycholysis. loss of thair

Ceptral Nervous System: akathisia, hallucinations, mood alterations, dream abnormalities, mental comt paresthesias

Urogenital System: Aysuria

Caronovascular Respiratory paipitations, dyspnea

Aypersensitivity positive ANA

Soecial Sesses hearing ingaicment
overDoOSAGE: In the event treatment for overdosage is required, the long plasma hail-life of piroxicam should be c ered. The absence of experience with acute overdosage precludes characterization of sequelae and recommendat

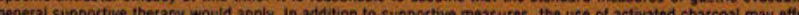

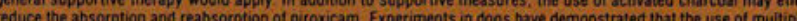

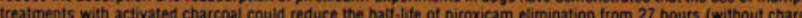
11 hours and raduce the systemic bioavailability of puroxicam by as much as $37 \%$ when activated charcoal is tiven

as 6 hours after administration of piroxicam.
ADMINISTRATION AND DOSAGE: Rheumatoid Arthritis, Osteoarthritis: It is recommended that FELOENE therapy tiated and maintained at a single daily dose of $20 \mathrm{mg}$. If desired. the daily dose may be divided

Uosage recommendations and indications for use in children have not been established

More detailed professional information available on request

C1982, Prizi

Reterences: 1 . Siegmeth $W$ : Serum concentrations of piroxicam in relation to its clinical effect in patients with chron yarthritis (trans). Wien Med Wochenschr 130:31-35, 1980.2. Tausch: Placebo-controlled study of piroxicam in the ment of meumatoid arthritis. Am J Med (report) 72.18-22. February 16. 1982. 